\title{
Locally targeted cytoprotection with dextran sulfate attenuates experimental porcine myocardial ischaemia/reperfusion injury
}

\author{
Yara Banz ${ }^{1}$, Otto M. Hess ${ }^{2}$, Simon C. Robson ${ }^{3}$, Daniel Mettler ${ }^{1}$, Pascal Meier $^{2}$, André Haeberli $^{1}$, \\ Eva Csizmadia ${ }^{3}$, Elena Y. Korchagina ${ }^{4}$, Nicolai V. Bovin ${ }^{4}$, and Robert Rieben ${ }^{1 *}$ \\ ${ }^{1}$ Department of Clinical Research, University of Bern, Murtenstrasse 31, 3010 Bern, Switzerland; ${ }^{2}$ Department of Cardiology, \\ Swiss Cardiovascular Center, University Hospital, Bern, Switzerland; ${ }^{3}$ Beth Israel Deaconess Medical Center, \\ Harvard University, Boston, MA, USA; and ${ }^{4}$ Shemyakin and Ovchinnikov Institute of Bioorganic Chemistry, Russian Academy \\ of Sciences, Moscow, Russia
}

Received 10 February 2005; revised 3 June 2005; accepted 27 June 2005; online publish-ahead-of-print 29 July 2005

\section{KEYWORDS}

Ischaemia reperfusion injury; Dextran sulfate; Cytoprotection; Endothelium

\begin{abstract}
Aims Intravascular inflammatory events during ischaemia/reperfusion injury following coronary angioplasty alter and denudate the endothelium of its natural anticoagulant heparan sulfate proteoglycan (HSPG) layer, contributing to myocardial tissue damage. We propose that locally targeted cytoprotection of ischaemic myocardium with the glycosaminoglycan analogue dextran sulfate (DXS, MW 5000) may protect damaged tissue from reperfusion injury by functional restoration of HSPG.

Methods and results In a closed chest porcine model of acute myocardial ischaemia/reperfusion injury (60 min ischaemia, 120 min reperfusion), DXS was administered intracoronarily into the area at risk 5 min prior to reperfusion. Despite similar areas at risk in both groups $(39 \pm 8 \%$ and $42 \pm 9 \%$ of left ventricular mass), DXS significantly decreased myocardial infarct size from $61 \pm 12 \%$ of the area at risk for vehicle controls to $39 \pm 14 \%$. Cardioprotection correlated with reduced cardiac enzyme release creatine kinase (CK-MB, troponin-I). DXS abrogated myocardial complement deposition and substantially decreased vascular expression of pro-coagulant tissue factor in ischaemic myocardium. DXS binding, detected using fluorescein-labelled agent, localized to ischaemically damaged blood vessels/myocardium and correlated with reduced vascular staining of HSPG.

Conclusion The significant cardioprotection obtained through targeted cytoprotection of ischaemic tissue prior to reperfusion in this model of acute myocardial infarction suggests a possible role for the local modulation of vascular inflammation by glycosaminoglycan analogues as a novel therapy to reduce reperfusion injury.
\end{abstract}

\section{Introduction}

Restoration of coronary blood flow after a period of prolonged ischaemia is a double-edged sword. Although reperfusion by means of thrombolysis or percutaneous coronary intervention $(\mathrm{PCl})$ is critical for tissue salvage, it aggravates ischaemic damage and instigates ischaemia/reperfusion (I/R) injury. ${ }^{1}$ Evidence suggests an important role for the complement system and its interactions with the endothelium in the pathogenesis of this type of tissue damage. ${ }^{2,3}$ Reperfusion greatly accelerates ischaemia-induced complement activation and deposition. ${ }^{4}$ The membrane attack complex directly stimulates synthesis of radical oxygen metabolites ${ }^{5}$ and promotes adhesion of neutrophils to the endothelium, ${ }^{6}$ and complement inhibition attenuates ischaemic tissue injury. ${ }^{7,8}$ In the clinical setting, complement

\footnotetext{
*Corresponding author. Tel: +4131632 9669; fax: +41316328837.
}

E-mail address: robert.rieben@dkf.unibe.ch inhibition using anti-C5 antibody treatment leads to a survival benefit after $\mathrm{PCl}^{9}$

Upon reperfusion after a prolonged period of ischaemia, the affected endothelium takes on a pro-coagulant and proinflammatory phenotype, with upregulation of pro-coagulant tissue factor (TF) and vascular adhesion molecules, as well as stimulation of cytokine production. ${ }^{10-12}$ Importantly, reperfusion appears to be critical for injury to the luminal surface coating of the endothelium, the glycocalyx. ${ }^{13,14}$ Indeed, inhibition of glycocalyx modifications upon reperfusion attenuates I/R damage. ${ }^{15}$ The glycocalyx, composed of a layer of glycoproteins, proteoglycans, and associated glycosaminoglycans, serves as a barrier to transvascular exchange of macromolecules and leukocyte adhesion ${ }^{16,17}$ and regulates the anticoagulant and anti-inflammatory properties of the endothelium by enhancing the activity of TF pathway inhibitor and antithrombin. ${ }^{18,19}$

In the current investigation, we studied the potential of low molecular weight dextran sulfate (DXS, MW 5000) to 
attenuate I/R injury in vivo. DXS, a sulfated polysaccharide and glycosaminoglycan analogue, inhibits the alternative, classical, and lectin complement pathways ${ }^{20-22}$ as well as the coagulation cascade. ${ }^{23}$ Furthermore, in vitro work in our laboratory has shown that DXS inhibits complementmediated endothelial damage following cleavage of luminal HSPGs by heparinase. ${ }^{22}$ For the current study, therefore, our working hypothesis was that cytoprotection targeted to ischaemically damaged endothelium and myocardium by DXS may attenuate tissue injury in acute myocardial infarction through modification of the local vascular environment.

\section{Methods}

Care and use of animals in the present study were in compliance with the Guide for the Care and Use of Laboratory Animals (NIH Publication No. 85-23, revised 1996).

\section{Experimental model}

Twenty large white pigs $(30 \pm 2 \mathrm{~kg})$ were pre-medicated with Ketamin/Xylazin, Midazolam, and Atropin, intubated, and mechanically ventilated with a Draeger respirator $\left(\mathrm{O}_{2} / \mathrm{N}_{2} \mathrm{O} 1: 3\right.$, Isoflurane 1-1.5 vol.\%). Central venous and arterial lines were introduced and a single bolus of unfractionated heparin (2500 IU) administered intravenously. Baseline values were recorded during a 30 -min preischaemic period.

The left anterior descending (LAD) artery was occluded just distally of the first diagonal branch with a semi-compliant overthe-wire $\mathrm{PCl}$ catheter (Concerto, Occam, the Netherlands; balloon length $10 \mathrm{~mm}$, diameter $2.5-3 \mathrm{~mm}$ ). The balloon was inflated to completely occlude the vessel (Encore $^{T M} 26$ inflation device, Boston Scientific, Ireland; used pressure 4-6 atm) for $60 \mathrm{~min}$. Localization of the balloon and state of inflation was controlled angiographically on a regular basis.

The 60 -min ischaemia period was followed by $2 \mathrm{~h}$ of reperfusion. Five minutes prior to reperfusion, $10 \mathrm{~mL}$ of DXS (MW 5000, Sigma Chemical Co., St Louis, MO, USA; $25 \mathrm{mg} / \mathrm{mL}$-this dose was chosen after extensive previous experience and in vitro and in vivo testing, $n=7$ ) or phosphate-buffered saline (PBS, vehicle control, $n=9$ ) was injected intracoronarily through the tip of the catheter into the area at risk (AAR) over a period of $2 \mathrm{~min}$. After $3 \mathrm{~min}$, the balloon was deflated to allow $2 \mathrm{~h}$ of reperfusion. After the reperfusion period, the balloon was re-inflated and $60 \mathrm{~mL}$ Evan's Blue injected intravenously. The animals were then sacrificed (intravenous bolus potassium chloride) and the heart excised for further analysis.

In four further experiments, fluorescein-labelled DXS (DXS-Fluo) was used instead of the normal, unlabelled substance. DXS-Fluo, possessing nearly identical biochemical properties as the unlabelled substance, was produced using fluorescein cadaverine (Molecular Probes Europe, Leiden, The Netherlands) as described earlier. ${ }^{22}$ Heart, lungs, liver, kidneys, and spleen were retrieved and tissue samples snap-frozen in Tissue-Tek OCT compound (Sakura Finetek Europe BV, Zoeterwoude, The Netherlands). These samples were used to analyse tissue distribution and binding of DXS.

ECG, mean arterial pressure (MAP), and left ventricular enddiastolic pressure (LVEDP) were recorded throughout with a Hewlett-Packard CMS patient monitor. Ejection fraction (EF) was determined angiographically and calculated using the area-length method according to Dodge. ${ }^{24}$

The experimenters, including the pathologists performing subsequent staining and histological/immunochemical analysis, were blinded with regard to treatment regimen. Randomization of the animals into two groups was done prior to the beginning of the experimental series, using a randomization code (PBS control animals $=0$, DXS animals $=1$ ) created by a random number generator (SAS, version 9.1.2, SAS Institute Inc., Cary, NC, USA). The $10 \mathrm{~mL}$ samples (PBS or DXS solution) were prepared according to the output of the randomization and stored at $-20^{\circ} \mathrm{C}$ until use. Randomization and sample preparation was performed by an independent laboratory technician. Before pre-medication, the corresponding vial was allocated to the pig (sequential number of $\mathrm{vial}=$ sequential number of the pig). All the 20 consecutively enlisted pigs were treated according to the aforementioned protocol. There were no dropouts throughout the entire procedure in any of the experiments. Sample size was determined from previous in vitro and in vivo work as well as experience, but not by formal sample size calculations.

In the case of ventricular fibrillation, a biphasic defibrillator $(150 \mathrm{~J})$ was used for cardioconversion.

\section{Infarct size}

The AAR was determined at the end of the experiment through $\angle A D$ re-occlusion and intravenous injection of $60 \mathrm{~mL}$ Evan's Blue ( $2 \%$ wt/vol. solution), leaving all but the AAR stained blue. The left ventricle was cut perpendicular to the septum from the apex to the base into $3 \mathrm{~mm}$ slices. Viable myocardium [viable ischaemic tissue (VIT)] from the AAR was stained bright red by incubating the slices in $1 \%$ triphenyl tetrazolium chloride (TTC, Sigma, $\mathrm{pH}$ 7.4) for $20 \mathrm{~min}$ at $37^{\circ} \mathrm{C}$. The tetrazolium dye forms a dark-red formazan complex in the presence of viable myocardium, which contains active dehydrogenases and cofactors. ${ }^{25}$ Infarcted tissue [necrotic ischaemic tissue (NIT)], however, remains unstained. The VIT was separated from NIT. All three tissue sections [Evan's Blue positive area not at risk (ANR), NIT, and VIT] were weighed. By definition, AAR is the sum of NIT and VIT. Data were expressed as total left ventricular mass (LVM) in grams, AAR in grams, AAR as per cent of LVM, NIT in grams, and NIT as per cent of AAR.

\section{Complement and coagulation}

Peripheral venous blood samples were collected (EDTA plasma/ serum), kept on ice until centrifugation $\left(1750 \mathrm{~g}\right.$ for $10 \mathrm{~min}$ at $4{ }^{\circ} \mathrm{C}$ ), and stored at $-70^{\circ} \mathrm{C}$ until further analysis.

Classical pathway complement activity was determined by standard $\mathrm{CH} 50$ assay with antibody-coated sheep erythrocytes. ${ }^{26}$ As a global parameter for the coagulation system, activated partial thromboplastin time (aPTT) was measured using Dade Actin FS reagent in a standard coagulation assay.

\section{Ischaemic markers}

The levels of troponin I and creatine kinase (CK-MB fraction) were determined by enzyme immunoassays (AXSYM microparticle enzyme immunoassay platform, Abbott Laboratories, Abbott Park, IL, USA). Both ELISAs were judged sensitive and specific for myocardial injury in pigs. Neither of the two parameters showed elevated levels at baseline or in healthy pigs.

\section{Histology and immunostaining}

Samples from ANR, NIT, and VIT were fixed in $4 \%$ buffered formaldehyde, paraffin-embedded, cut into $3 \mu \mathrm{m}$ sections, and stained with haematoxylin-eosin for histological evaluation.

Five micrometre sections were cut from all snap-frozen tissue samples, air-dried, acetone fixed, hydrated, and labelled using a two/three-step indirect immunofluorescence technique. The antibodies used were rabbit anti-human C1q, C3c/C3b, and C4c (Dako); mouse monoclonal anti-human C7 (Quidel, Santa Clara, CA, USA); and rat monoclonal anti-human HSPG (Abcam Limited, Cambridge, UK). Cross-reactivity with the respective porcine antigens was verified. Secondary antibodies were goat anti-rabbit $\operatorname{lgG}(\mathrm{H}+\mathrm{L})$-FITC (Southern Biotechnology Associated, Birmingham, AL, USA), rabbit anti-mouse Ig-FITC (Dako), and biotinylated goat anti-rat IgG (Southern Biotechnology), followed by streptavidinFITC (Amersham Pharmacia Biotech). Immunohistochemical staining 
for TF with polyclonal rabbit anti-TF antibody was carried out as reported previously. ${ }^{27,28}$

\section{Statistics}

Data from TTC staining (AAR/LVM and NIT/AAR in per cent) were compared between groups by use of one-way analysis of variance (ANOVA). Results for the DXS and PBS control groups for all other parameters were compared by use of two-way repeated-measures ANOVA with time and treatment as fixed factors. Subject number was included as random. Effects of interest were overall betweengroup differences and interaction between group and time. The assumption of the sphericity of our data was analysed by Mauchly's test and corrections were made where appropriate (GreenhouseGeisser). In the case of a significant $P$-value in the overall ANOVA with respect to between-group differences or group-time interaction, post-hoc analyses were performed. $P$-values were corrected for multiple testing by Bonferroni correction. For all analyses, $P$-values were two-sided and differences were considered to be statistically significant with a $P$-value of $<0.05$. SAS Version 9.1 .2 (SAS Institute Inc., Cary, NC, USA) and SPSS Version 12.0.1 (SPSS Inc., Chicago, IL, USA) software were used for all analyses. Data, unless otherwise specified, are presented as average \pm standard deviation in text and figures.

(A)

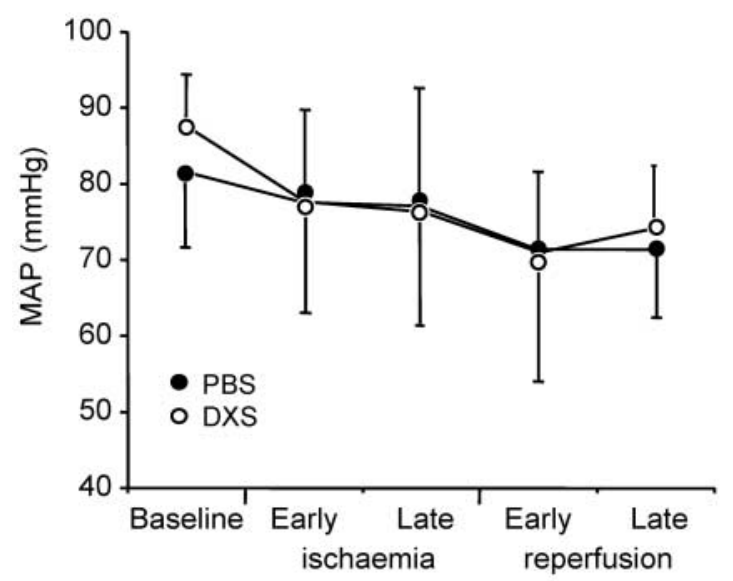

(C)

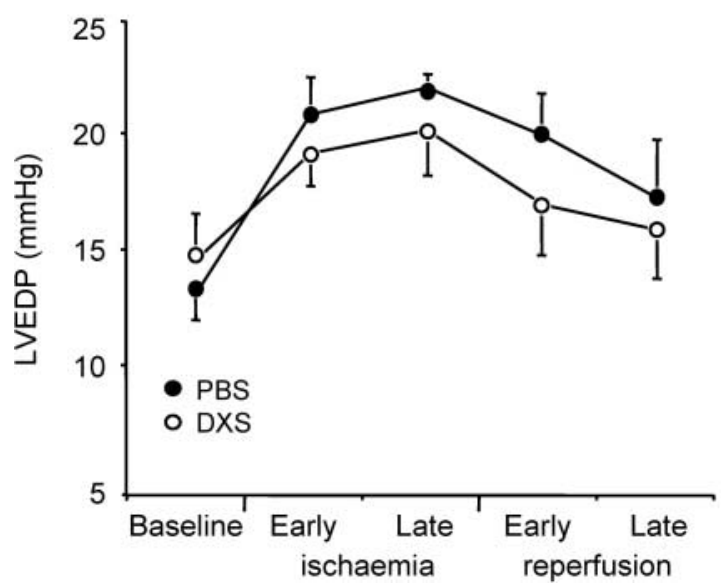

\section{Results}

\section{Haemodynamic variables}

During the experiments, there were no significant differences in MAP between the two groups $(P=0.547$ for between-group differences, $P=0.253$ for group-time interaction). MAP dropped in both groups during late occlusion and during the early reperfusion period (Figure 1A). In both groups, heart rate decreased during ischaemia and increased again after the onset of reperfusion, with no significant differences noted $(P=0.937$ for between-group differences, $P=0.617$ for group-time interaction, Figure $1 B$ ). Baseline LVEDP and the observed increase during occlusion of the LAD were comparable in both groups. LVEDP rapidly decreased upon restoration of coronary blood flow in both groups. Though not statistically significant, LVEDP showed better recovery in the DXS-treated animals when compared with PBS controls until the end of the $2 \mathrm{~h}$ reperfusion phase $(P=0.190$ for betweengroup differences, $P=0.456$ for group-time interaction, Figure $1 \mathrm{C})$. EF dropped during the $1 \mathrm{~h}$ ischaemia period in both groups and did not substantially recover until the end of the $2 \mathrm{~h}$ of reperfusion $(P=0.731$ for between-group

(B)

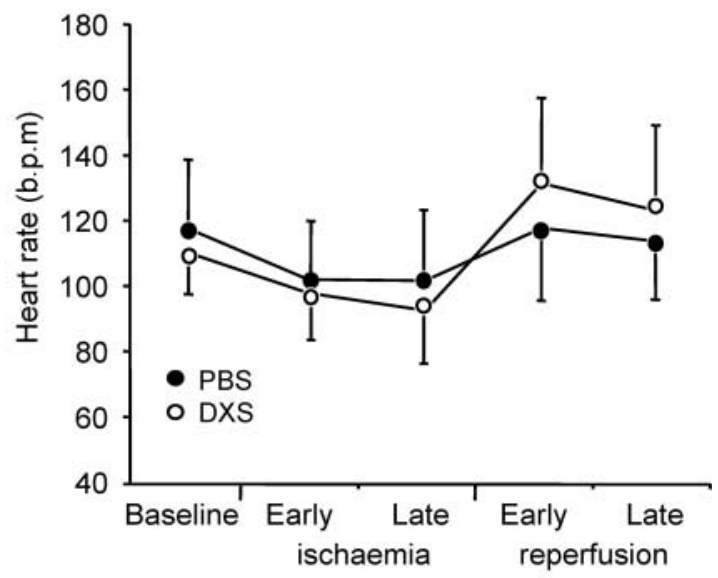

(D)

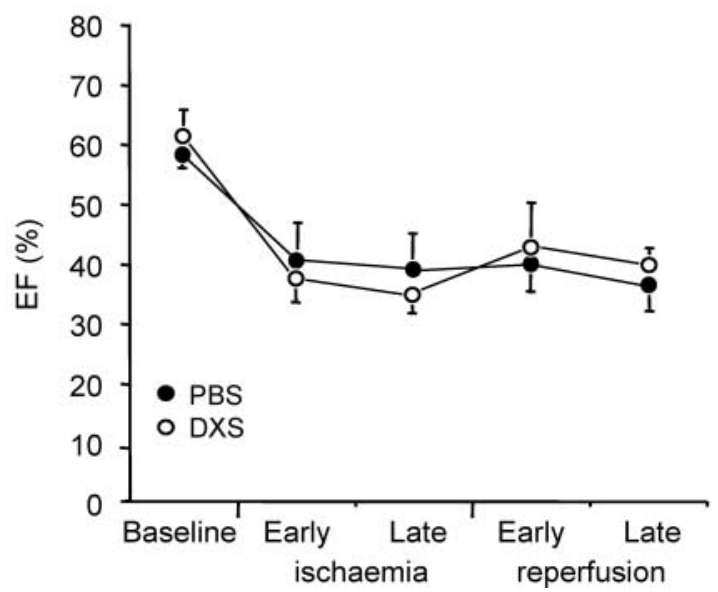

Figure 1 Monitoring of MAP $(A)$ and heart rate $(B)$ before occlusion (baseline), during early (first half hour) and late (second half hour) ischaemia, and during early (first hour) and late (second hour) reperfusion phase. No significant differences in MAP or heart rate between groups. LVEDP showed better recovery in the DXS group during reperfusion, particularly in the first hour $(C)$. Drop in EF during ischaemia, with no substantial recovery during $2 \mathrm{~h}$ of reperfusion and no significant inter-group differences $(D)$. Data are mean \pm standard deviation. 
differences, $P=0.410$ for group-time interaction, Figure 1D).

\section{Soluble complement and coagulation parameters}

Intracoronary DXS administration did not systemically inhibit the classical complement pathway, as determined by $\mathrm{CH} 50$ test. $\mathrm{CH} 50$ baseline values were comparable in both groups. No significant changes were observed during the course of the experiment $(P=0.970$ for between-group differences, $P=0.548$ for group-time interaction, Figure $2 A$ ).

DXS transiently inhibited the coagulation system with prolongation of aPTT from $50 \pm 13 \mathrm{~s}$ during occlusion to $110 \pm 51 \mathrm{~s}$ at $5 \mathrm{~min}$ of reperfusion. Values normalized again to $50 \pm 7 \mathrm{~s}$ at the $2 \mathrm{~h}$ point of reperfusion. No prolongation of aPTT was noted in the PBS control group ( $P=0.098$ for between-group differences, $P=0.163$ for group-time interaction, Figure $2 B$ ).

\section{Plasma creatine kinase and troponin I}

Cellular damage evaluated by levels of creatine kinase (CK-MB, Figure $3 A$ ) and troponin I (Figure 3B) increased upon reperfusion in both the DXS and PBS groups. The release of CK-MB, and particularly troponin I, was more pronounced in the PBS group. Inter-group difference, although clearly visible, did not reach statistical significance until the end of the experiment after $2 \mathrm{~h}$ of reperfusion $(P=0.855$ for between-group differences, $P=0.256$ for group-time interaction for CK-MB; $P=0.410$ for between-group differences, $P=0.148$ for group-time interaction for troponin I).

\section{Infarct size}

The myocardial AAR, expressed as percentage of the LVM, was not significantly different in the two groups (mean $39 \pm 8 \%$ /median $35.5 \%$ in PBS controls, mean $42 \pm 9 \%$ / median $40.0 \%$ in DXS-treated animals; $P=0.177$, Figure $3 C$ ). Corresponding wet weights were $33.1 \pm 10.9$ and $34.8 \pm 12.6 \mathrm{~g}$, respectively. However, infarct size (NIT) was significantly lower in the DXS-treated animals when compared with the PBS controls (NIT expressed as per cent of AAR: $39 \pm 14 \% / 38.4 \%$ vs. $61 \pm 12 \% / 66.5 \%, P=0.003$, Figure $3 D$ ). The respective wet weights were $12.6 \pm 4.8 \mathrm{~g}$ in the DXS group and $19.9 \pm 6.5 \mathrm{~g}$ in the PBS controls. Reduction of infarct size in space was not uniform in the individual experiments. In four DXS experiments, 'additional' live tissue was distributed in a patchy fashion within the area of necrosis, whereas in the other three DXS experiments, infarct size reduction resulted from retraction of infarct borders. An example of TTC staining result with corresponding histology is shown in Figure 4.

\section{Histology: infarct morphology}

Typical signs of reperfusion damage with contraction bands and coagulation necrosis were observed in the infarcted area (NIT; Figure 4, right panel) from the DXS and PBS groups, with no substantive differences between groups. Gross haemorrhage was detected in one animal of each group. Foci of microscopic haemorrhagic infarction were equally detected in both groups ( $n=2$ per group). Samples from VIT (Figure 4, left panel) in both groups only focally revealed minimal histological signs of ischaemia (wavy fibres). ANR samples showed normal histological findings.

\section{Complement deposition, HSPG staining, and TF expression}

Figure 5 summarizes the data of complement deposition, HSPG staining, and TF expression. Samples from all experiments were stained and graded: $0=$ no staining, $1=$ minimal focal or diffuse staining, $2=$ moderately strong focal or diffuse staining, 3 = extensive focal or diffuse staining. Normal tissue samples from healthy swine (not shown) and ANR were used as controls.

Complement deposition (C1q, C4c, C3c/C3b, and C7) in the NIT (in vasculature and on damaged myocytes) was markedly decreased in the DXS-treated animals when compared with PBS controls. Staining for complement deposition was essentially negative in myocardium of healthy swine and only traces of complement were detected in samples from the ANR of both groups (Figure 6A).

Ischaemic tissue, particularly NIT, showed less positivity for HSPG staining when compared with non-ischaemic samples. Inter-group differences were difficult to detect, as positive staining was not limited to vascular HSPG (Figure 6B).
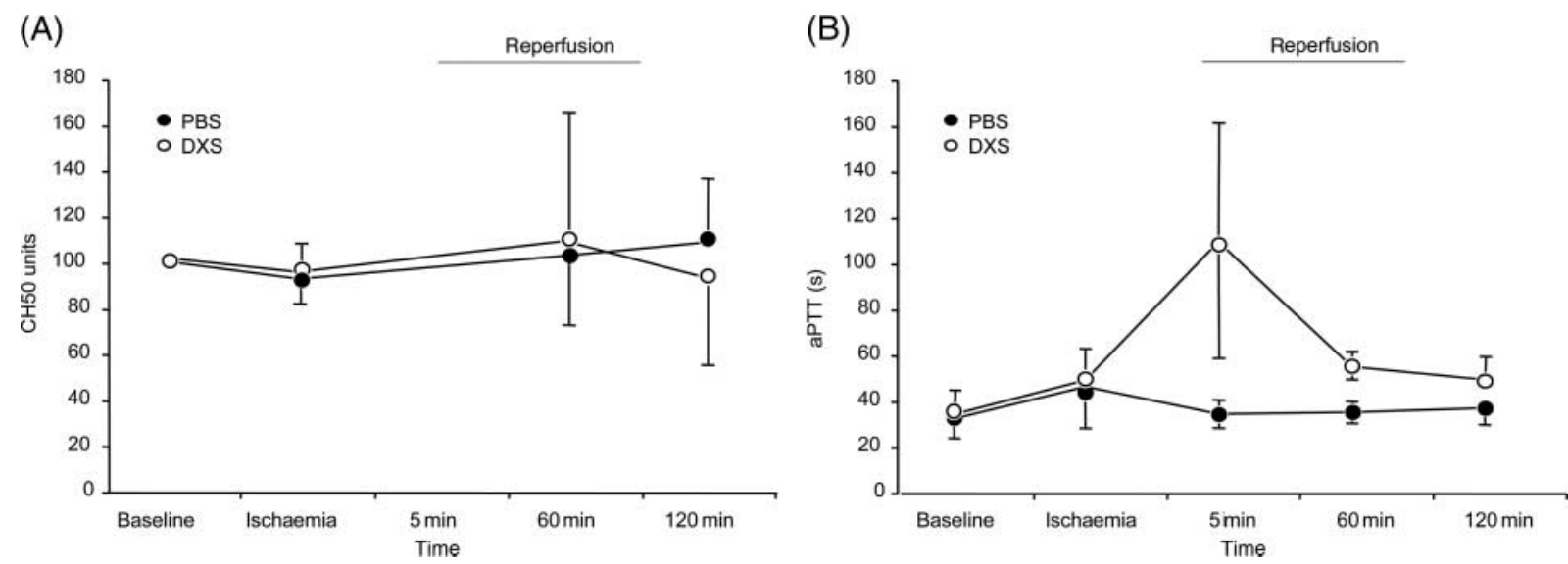

Figure 2 No systemic inhibition of the classical complement pathway in either of the experimental groups as measured in CH50 test ( $A$ ). Transient inhibition of the coagulation system with DXS, with aPTT prolongation at $5 \mathrm{~min}$ of reperfusion and normalization after $2 \mathrm{~h}$ of reperfusion. No prolongation of aPTT in the PBS control group $(B)$. Data are mean \pm standard deviation. 
(A)

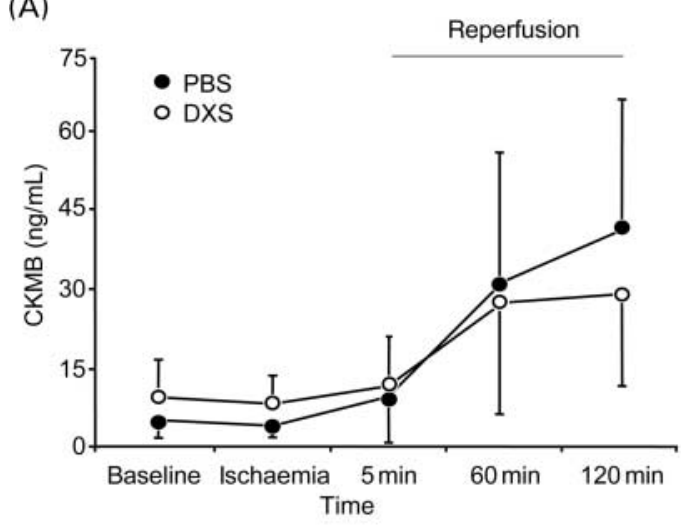

(C)

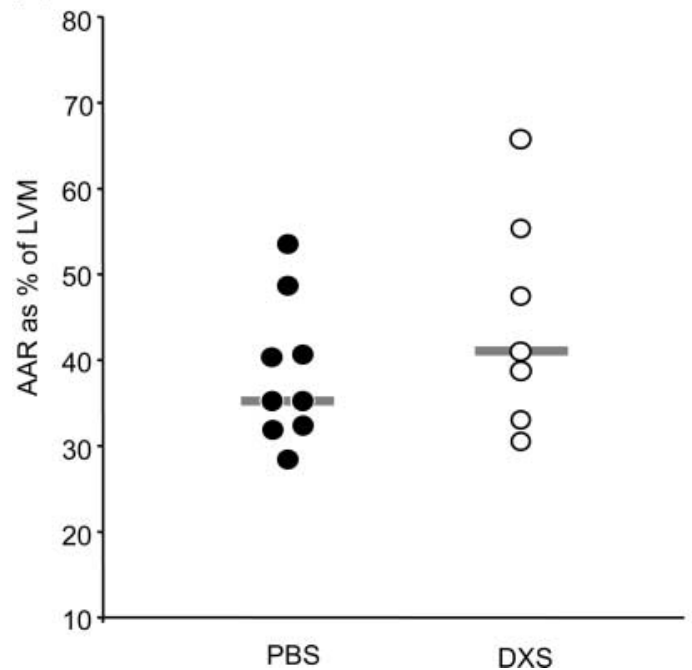

(B)

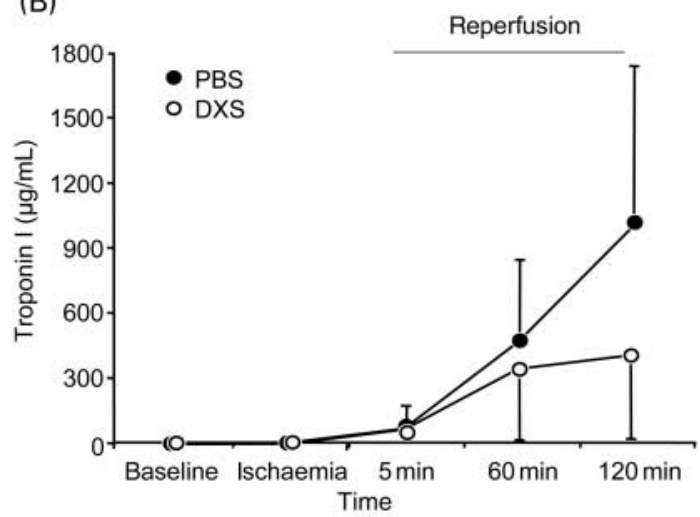

(D)

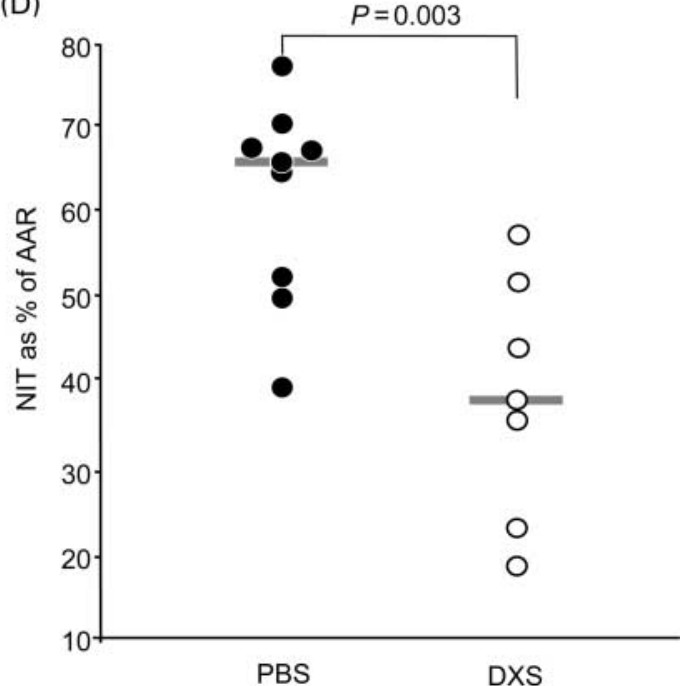

Figure 3 Enzymatic infarct size measured by creatine kinase (CK-MB fraction) and troponin I $(A$ and $B)$ in peripheral venous blood samples. Data are mean \pm standard deviation. Ratios of AAR to LVM and NIT to AAR ( $C$ and $D$ ). Individual values and medians (bars) are shown. AAR was comparable in both groups. DXS treatment significantly reduced infarct size when compared with PBS controls (mean $39 \pm 14 \%$, median $38.4 \%$ with DXS, vs. $61 \pm 12 \% / 66.5 \%$, with PBS $P=0.003$ ).

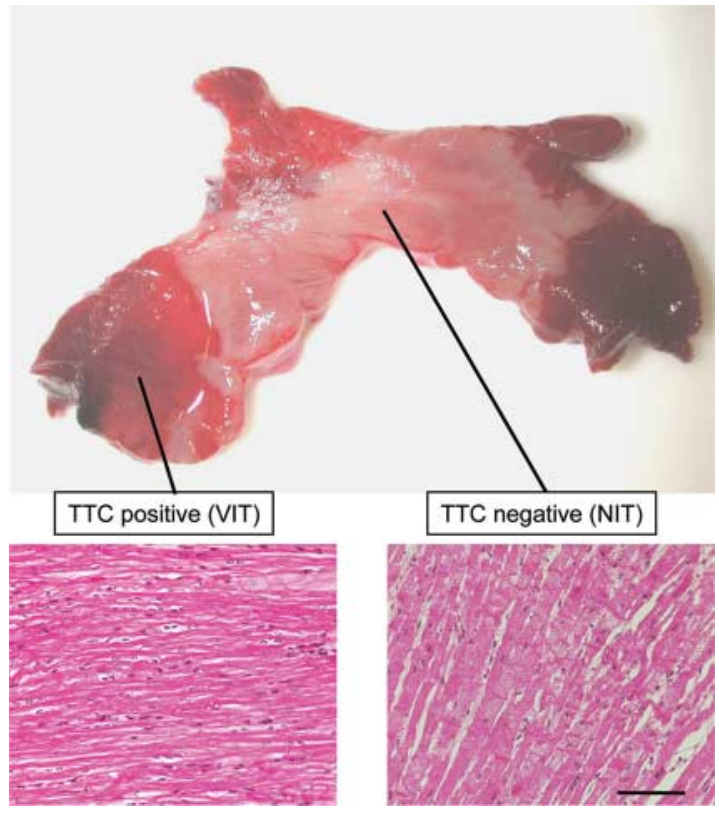

Figure 4 TTC staining of representative section of left ventricle (PBS control experiment) with corresponding histology from TTC red, viable ischaemic tissue (VIT) and TTC unstained, necrotic ischaemic tissue (NIT). Haematoxylineosin staining (scale bar: $50 \mu \mathrm{m}$ ).
TF expression was decreased in blood vessels within the NIT from DXS-treated animals when compared with PBS controls. In this latter group, upregulation of TF within the injured vasculature (mainly endothelial surface) was associated with the infarct areas. Weak focal TF staining was noted in select vessels obtained from the ANR and VIT, with no differences between the two groups. Minimal expression of TF was observed within native cardiac vasculature of healthy swine (Figure 6C).

\section{Binding of DXS-Fluo}

DXS-Fluo was used in four experiments to reveal adherence of DXS to the tissue. Binding of DXS-Fluo was detected specifically in the AAR (NIT and VIT regions) on the inner lining of small and large blood vessels and focally in strongly contracted myocytes (Figure 6D). DXS-Fluo binding was detected neither in the ANR of the heart nor in lungs, liver, kidney, or spleen in any of the samples taken from the four experiments.

\section{Discussion}

In the present study, we report that local, intracoronary application of the glycosaminoglycan analogue DXS, just 

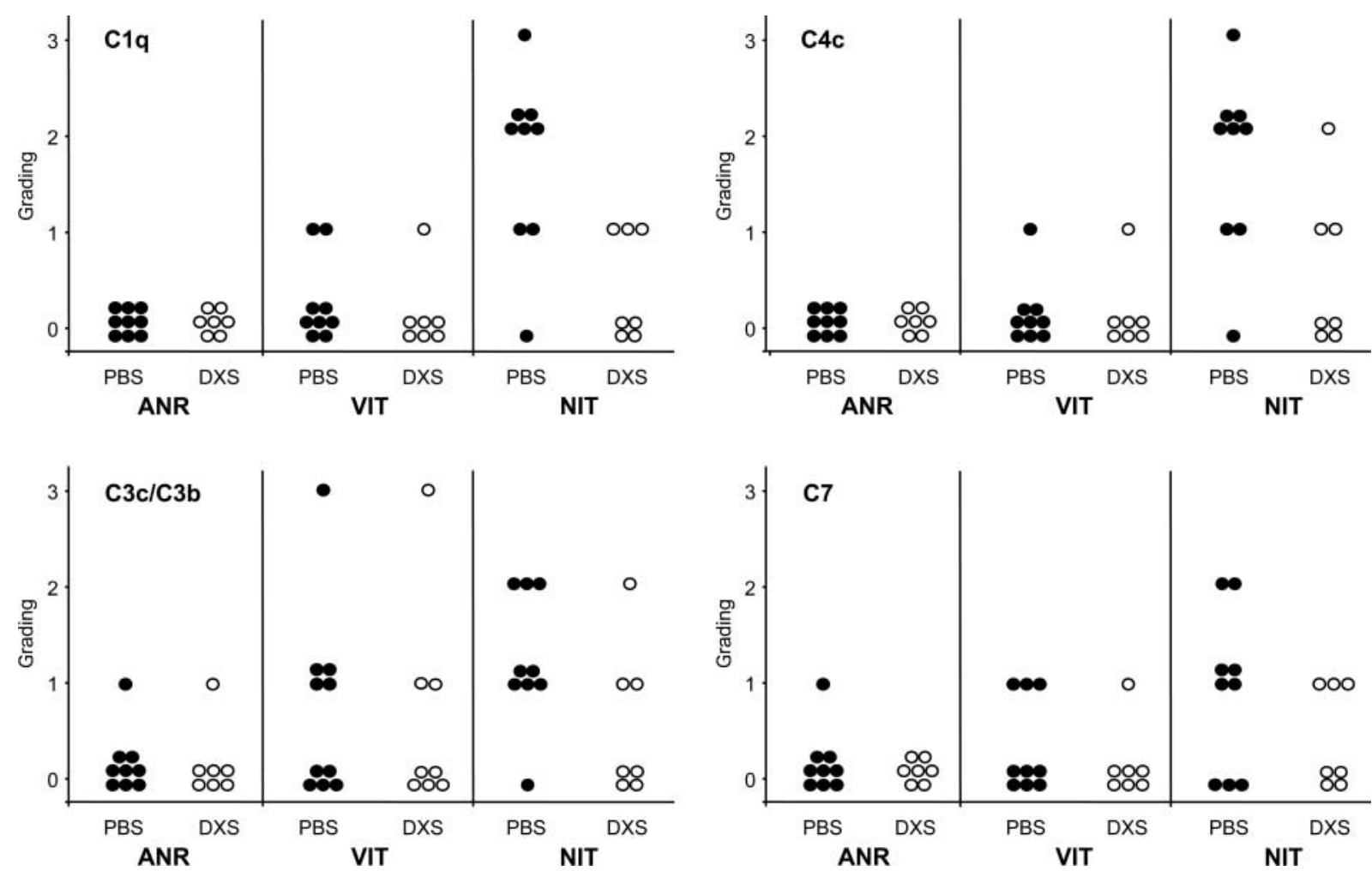

Figure 5 Grading of complement staining. Four samples per area and experiment were graded with respect to intensity for complement staining. Every point represents the average of all evaluations for one experiment. Bars indicate median values. $0=$ essentially no staining, $1=$ minimal focal or diffuse staining, $2=$ moderately strong focal or diffuse staining, and $3=$ extensive diffuse staining [Saline controls (PBS, $n=9)$ and DXS $(n=7)$ ].

prior to reperfusion, can protect ischaemic vasculature, as well as the surrounding myocardial tissue from the consequences of reperfusion injury. This pharmacological cardioprotection correlated directly with specific binding of DXS-Fluo to ischaemically damaged vasculature and surrounding myocardium.

Glycosaminoglycans and their analogues, such as heparin and pentosan sulfate, have previously been used systemically to attenuate I/R injury. Non-anticoagulant $N$-acetyl heparin and sulodexide, a mixture of glycosaminoglycans with limited anticoagulant activity, proved as effective as normal heparin. ${ }^{29,30}$ It may be complement inhibition rather than systemic anticoagulation providing protection, as well as the glycosaminoglycans' ability to rapidly associate with the endothelium. ${ }^{31}$

Alterations of the endothelial glycocalyx have been described to contribute to I/R injury, ${ }^{13,14}$ and inhibition of these glycocalyx modifications by adenosine A2A receptor activation attenuates tissue damage. ${ }^{15}$ From our own previous in vitro work, we know that DXS associates with endothelial cells after cleavage of glycocalyx HSPGs by heparinase, and that this binding correlates with complement inhibition. ${ }^{22}$ Presently, we could localize DXS binding to ischaemically damaged blood vessels using DXSFluo. This correlated with reduced vascular HSPG staining observed in these areas.

Contribution of the complement system to myocardial I/R injury has been documented early on. ${ }^{32}$ Inhibition of the complement system with fluid-phase ${ }^{33}$ or membranetargeted inhibitors ${ }^{34}$ has proved successful both in animal models and in clinical settings. ${ }^{9,35}$ In our model, binding of
DXS within the ischaemic area correlated with attenuation of deposition of $\mathrm{C} 1 \mathrm{q}$ as well as C4b/c and C3b/c. C1q selectively accumulates in ischaemic-reperfused myocardium, concentrations correlating reciprocally with regional myocardial blood flow. ${ }^{36}$ Indeed, fixation of C1q to subcellular fractions of myocardial cells, released upon ischaemic damage, activates the complement cascade and stimulates infiltration of polymorphonuclear leukocytes. ${ }^{37}$ However, sequential activation and release of C5a, TGF-beta 1, and MCP-1 may also promote myocardial healing following reperfusion by monocyte recruitment. ${ }^{38}$ DXS also reduced deposition of the terminal complement complex (TCC), detected as C7. TCC may directly be involved in complement-induced injury in $\mathrm{I} / \mathrm{R},{ }^{3}$ inhibition of its formation possibly prove critical to preserving EC integrity. Whether DXS, known to inhibit the lectin pathway in vitro, ${ }^{22}$ was likewise inhibitory in this model is unclear, as antibodies against porcine mannose-binding lectin (MBL) were not available to us. A role for the lectin pathway in I/R injury is emerging; ${ }^{39}$ selective inhibition of this pathway using monoclonal antibodies against MBL has proved beneficial in myocardial I/R injury in rats. ${ }^{40}$ Whether attenuation of the local pro-inflammatory environment pre-disposes to reduced complement activation and tissue deposition or whether complement inhibition precludes induction of vascular inflammation remains to be studied.

Reperfusion damage not only renders the endothelium pro-inflammatory, but also pro-coagulant. Healthy endothelium maintains cell-surface anticoagulation by expressing thrombomodulin ${ }^{41}$ and heparan sulfates, which enhance the activity of TF pathway inhibitor and antithrombin. ${ }^{18,19}$ 
(A)

C1q

$\mathrm{C} 4 \mathrm{c}$

$\mathrm{C} 3 \mathrm{cl}$

$\mathrm{C} 3 \mathrm{~b}$

C7

(B)

HSPG

(C)
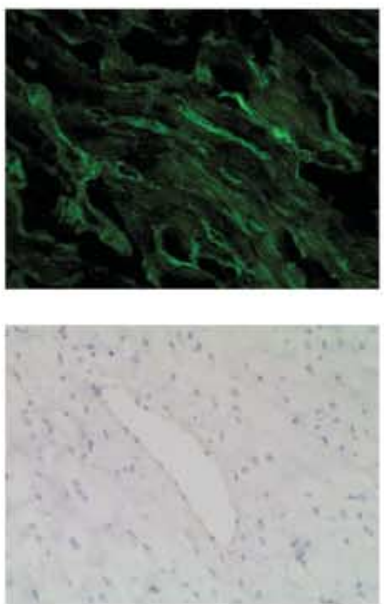

(D)

TF

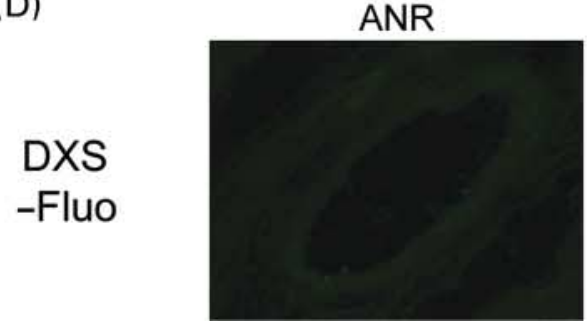

NIT, PBS group
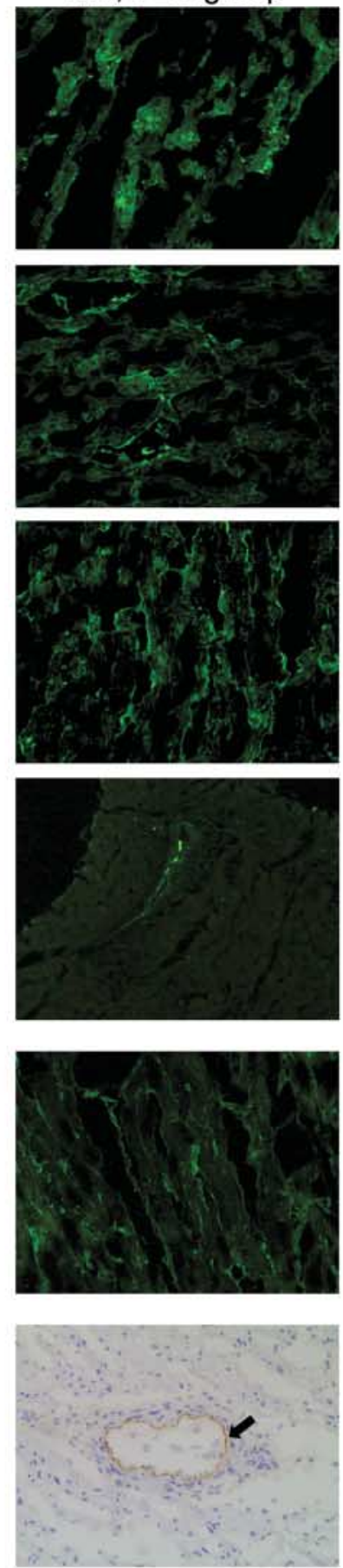

Ischaemic area

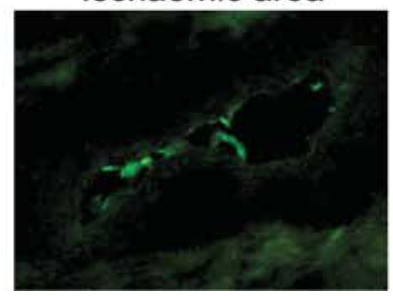

NIT, DXS group
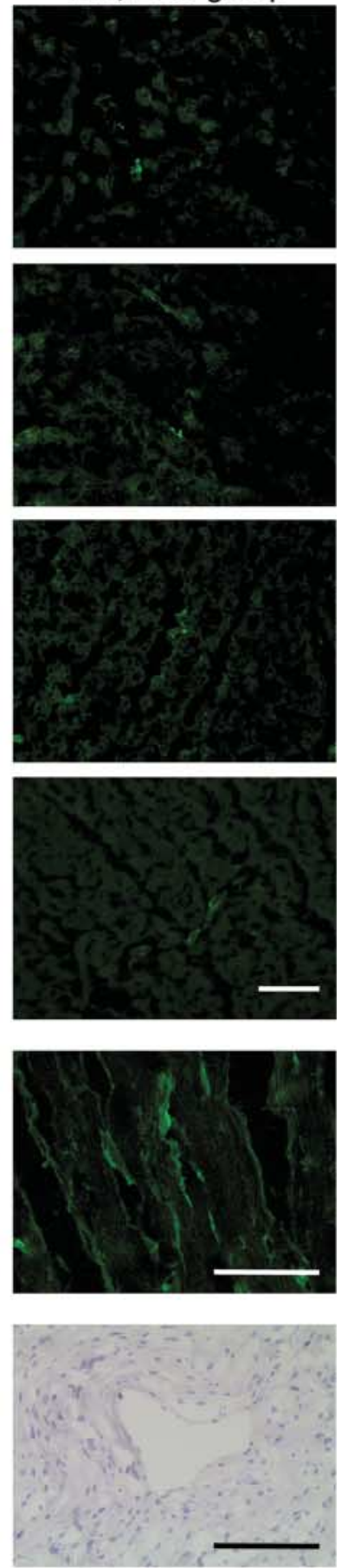

Ischaemic area

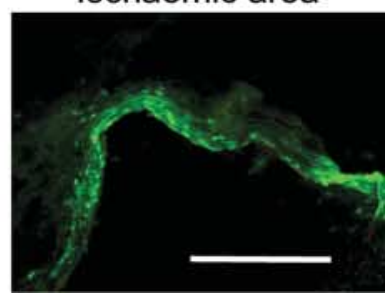

Figure 6 Effect of DXS treatment on deposition of complement on pig myocardium $(A)$. Staining for HSPG on pig myocardium $(B)$ and TF in myocardial blood vessels $(C)$. Brown staining (black arrow) reveals TF expression, especially in PBS controls. Specific binding of DXS-Fluo to inner lining of blood vessels and focally to strongly contracted myocytes in the infarcted area. No staining in blood vessels from the ANR $(D)$. All images are representative of at least four scored sections per area (ANR, NIT, VIT) per experiment. Scale bars: $50 \mu \mathrm{m}$. 
Heparinase treatment abolishes this endothelial surface property. ${ }^{42}$ Functionally replacing the shed HSPG layer by a glycosaminoglycan analogue, such as DXS, may help restore the essential property of 'surface regulation' of the coagulation system. Successful cell surface-targeted anticoagulation in systemic infection and inflammation has been described recently. ${ }^{43}$ Here, the ability of surfacebound DXS to modify the local pro-coagulant environment and its effect on inhibition of TF upregulation was studied. DXS treatment clearly decreased vascular TF expression within the infarcted area when contrasted to PBS controls. This suggests TF induction on vascular endothelium upon reperfusion, or recruitment with the sequestration of circulating microparticles, ${ }^{44}$ subsequent to exposure to complement components, reactive oxygen species, and activated leukocytes. Exposure of TF on activated EC (and monocyte/macrophages) to circulating coagulation factors activates the extrinsic pathway of clotting via binding to factor $\mathrm{VII},{ }^{45}$ generating a fibrin clot. Limiting EC damage and TF expression by DXS may be pivotal in reducing tissue damage by attenuating intravascular thrombosis and inflammation. Indeed, functional inhibition of TF with a monoclonal anti-TF antibody or human recombinant, active site-blocked activated factor VII, significantly reduced infarct size in rabbit models of MI. $^{12,46}$

Intracoronary application of DXS also led to a transient and mild inhibition of the fluid-phase coagulation system (aPTT prolongation). However, a therapeutically relevant prolongation of aPTT was only observed immediately after DXS injection, suggesting that fluid-phase, systemic inhibition of the coagulation system is of minor importance in our model. Indeed, intracoronary infusion of fractionated heparin, a glycosaminoglycan analogue with more potent anticoagulant but lower complement-inhibitory properties than DXS, did not reduce infarct size $(n=2$, results not shown). On the contrary, infarcts were grossly haemorrhagic and tissue exceedingly friable.

DXS did not significantly affect haemodynamic parameters within the observed reperfusion period. In models of myocardial I/R injury, some authors describe beneficial effects on haemodynamics using complement inhibitors, ${ }^{47}$ whereas others note no advantages. ${ }^{48}$ Clinical investigations imply that early functional assessment after Q-wave myocardial infarction has limited ability to predict recovery of ventricular function. Creatine kinase levels, however, were independently predictive of recuperation of function. ${ }^{49}$ Within $2 \mathrm{~h}$ of reperfusion, therefore, potentially favourable effects of DXS on myocardial performance may still have been masked by myocardial stunning.

Increasing clinical evidence suggests that time-to-treatment significantly determines post-Ml outcome following coronary intervention, ${ }^{50,51}$ confirming results from animal models, which show that duration of coronary occlusion is a main determinant of infarct size. ${ }^{52,53}$ The contribution, therefore, of reperfusion to irreversible cell injury decreases with increasing ischaemia time. In our model, the extent of irreversible cell damage after only $1 \mathrm{~h}$ ischae$\mathrm{mia} / 2 \mathrm{~h}$ reperfusion is rather high. However, this is in accordance with previously published work using pig models. ${ }^{7,47}$ The near absence of collaterals in pigs may explain such extensive necrosis. ${ }^{54}$ To study the effects of DXS on reducing reperfusion-induced damage, we chose to limit ischaemia time to $1 \mathrm{~h}$. The effect of DXS or similar substances in the clinical setting, although no less valuable, may be less dramatic as long as time-to-treatment is not cut back. Furthermore, treatment suitability of such substances needs to be evaluated with more clinically applicable, comprehensive therapeutic intervention and longer follow-ups.

TTC staining, used to differentiate between viable and necrotic tissue, allows sensitive and specific determination of infarct size, ${ }^{55}$ but may require sufficient reperfusion for accurate assessment. ${ }^{56}$ Although propidium iodide may prove more accurate for shorter reperfusion, ${ }^{57}$ it cannot discriminate between areas of no reflow and viable myocardium. ${ }^{56}$ TTC staining validity for 120 min reperfusion in pigs has already been confirmed, where viable TTC positive islets within TTC negative necrotic tissue correlated with electron microscopy findings. ${ }^{8}$

Our results support the hypothesis that cytoprotection by DXS, targeted to an ischaemically altered and damaged endothelium, can substantially decrease I/R injury. This cytoprotection correlates with the association of DXS with the damaged endothelium and reduced local complement deposition. However, the mechanisms providing the beneficial effects in this model are likely to be multidimensional, as the glycocalyx is crucial for endothelial function. Other endothelium-related mechanisms such as protection against myocardial oedema ${ }^{58}$ or inhibition of leukocyte adhesion ${ }^{59}$ may be involved. The exact nature of the protective effect of DXS remains to be investigated in greater detail. Nonetheless, we believe that the concept of targeted cytoprotection of ischaemic tissue using substances such as DXS, which locally modulate the pro-inflammatory and procoagulant environment, may offer an attractive novel strategy for the development of a clinically significant therapy for reperfusion injury, complementing standard therapy.

\section{Acknowledgements}

This study was supported by the Swiss Heart Foundation, the Swiss National Science Foundation (3200B0-104228, 7SUPJ062207), and the Russian Academy of Science programme 'Physico-chemical biology'. Y.B. is a recipient of an MD-PhD grant of the Swiss Academy of Medical Sciences. The authors wish to thank Professor Thomas Schaffner and Dr Andreas Kappeler for invaluable support and Olgica Beslac as well as Trinh Cung for excellent technical assistance, and Dr Gert Printzen and his team for help with the CK-MB and troponin I measurements. Anti-TF antibodies were a kind gift of Professor Yale Nemerson, Mount Sinai, School of Medicine, New York.

\section{References}

1. Matsumura K, Jeremy RW, Schaper J, Becker LC. Progression of myocardial necrosis during reperfusion of ischemic myocardium. Circulation 1998; 97:795-804.

2. Kilgore KS, Friedrichs GS, Homeister JW, Lucchesi BR. The complement system in myocardial ischaemia/reperfusion injury. Cardiovasc Res 1994; 28:437-444.

3. Zhou W, Farrar CA, Abe K, Pratt JR, Marsh JE, Wang Y, Stahl GL, Sacks SH. Predominant role for $\mathrm{C} 5 \mathrm{~b}-9$ in renal ischemia/reperfusion injury. $J$ Clin Invest 2000;105:1363-1371.

4. Mathey D, Schofer J, Schafer HJ, Hamdoch T, Joachim HC, Ritgen A, Hugo F, Bhakdi S. Early accumulation of the terminal complement-complex in the ischaemic myocardium after reperfusion. Eur Heart $J$ 1994; 15:418-423.

5. Adler S, Baker PJ, Johnson RJ, Ochi RF, Pritzl P, Couser WG. Complement membrane attack complex stimulates production of reactive oxygen metabolites by cultured rat mesangial cells. J Clin Invest 1986;77: 762-767. 
6. Kilgore KS, Park JL, Tanhehco EJ, Booth EA, Marks RM, Lucchesi BR. Attenuation of interleukin-8 expression in C6-deficient rabbits after myocardial ischemia/reperfusion. J Mol Cell Cardiol 1998;30:75-85.

7. Amsterdam EA, Stahl GL, Pan HL, Rendig SV, Fletcher MP, Longhurst JC. Limitation of reperfusion injury by a monoclonal antibody to C5a during myocardial infarction in pigs. Am J Physiol 1995;268:H448-H457.

8. Horstick G, Berg O, Heimann A, Gotze O, Loos M, Hafner G, Bierbach B, Petersen S, Bhakdi S, Darius H, Horstick M, Meyer J, Kempski O. Application of $\mathrm{C} 1$-esterase inhibitor during reperfusion of ischemic myocardium: dose-related beneficial versus detrimental effects. Circulation 2001;104:3125-3131.

9. Granger CB, Mahaffey KW, Weaver WD, Theroux P, Hochman JS, Filloon TG, Rollins S, Todaro TG, Nicolau JC, Ruzyllo W, Armstrong PW. Pexelizumab, an anti-C5 complement antibody, as adjunctive therapy to primary percutaneous coronary intervention in acute myocardial infarction: the COMplement inhibition in Myocardial infarction treated with Angioplasty (COMMA) trial. Circulation 2003;108:1184-1190.

10. Golino P, Ragni M, Cirillo P, Avvedimento VE, Feliciello A, Esposito N, Scognamiglio A, Trimarco B, laccarino $G$, Condorelli $M$, Chiariello $M$, Ambrosio $G$. Effects of tissue factor induced by oxygen free radicals on coronary flow during reperfusion. Nat Med 1996;2:35-40.

11. Koo DD, Welsh KI, Roake JA, Morris PJ, Fuggle SV. Ischemia/reperfusion injury in human kidney transplantation: an immunohistochemical analysis of changes after reperfusion. Am J Pathol 1998;153:557-566.

12. Erlich JH, Boyle EM, Labriola J, Kovacich JC, Santucci RA, Fearns C, Morgan EN, Yun W, Luther T, Kojikawa O, Martin TR, Pohlman TH, Verrier ED, Mackman N. Inhibition of the tissue factor-thrombin pathway limits infarct size after myocardial ischemia-reperfusion injury by reducing inflammation. Am J Pathol 2000;157:1849-1862.

13. Czarnowska E, Karwatowska-Prokopczuk E. Ultrastructural demonstration of endothelial glycocalyx disruption in the reperfused rat heart. Involvement of oxygen free radicals. Basic Res Cardiol 1995;90: 357-364.

14. Mulivor AW, Lipowsky HH. Inflammation and ischemia induced shedding of the venular glycocalyx. Am J Physiol Heart Circ Physiol 2004; 286:H1672-1680.

15. Platts SH, Linden J, Duling BR. Rapid modification of the glycocalyx caused by ischemia-reperfusion is inhibited by adenosine A2A receptor activation. Am J Physiol Heart Circ Physiol 2003;284:H2360-H2367.

16. Ihrcke NS, Wrenshall LE, Lindman BJ, Platt JL. Role of heparan sulfate in immune system-blood vessel interactions. Immunol Today 1993;14: 500-505.

17. Constantinescu AA, Vink H, Spaan JA. Endothelial cell glycocalyx modulates immobilization of leukocytes at the endothelial surface. Arterioscler Thromb Vasc Biol 2003;23:1541-1547.

18. Hirsh J, Levine MN. Low molecular weight heparin. Blood 1992;79:1-17.

19. Ho G, Broze GJ Jr, Schwartz AL. Role of heparan sulfate proteoglycans in the uptake and degradation of tissue factor pathway inhibitorcoagulation factor Xa complexes. J Biol Chem 1997;272:16838-16844.

20. Meri S, Pangburn MK. Regulation of alternative pathway complement activation by glycosaminoglycans: specificity of the polyanion binding site on factor H. Biochem Biophys Res Commun 1994;198:52-59.

21. Wuillemin WA, te Velthuis $H$, Lubbers $Y T$, de Ruig CP, Eldering E, Hack CE. Potentiation of $\mathrm{C} 1$ inhibitor by glycosaminoglycans: dextran sulfate species are effective inhibitors of in vitro complement activation in plasma. J Immunol 1997;159:1953-1960.

22. Laumonier T, Walpen A, Maurus C, Mohacsi PJ, Matozan K, Korchagina E, Bovin N, Vanhove B, Seebach JD, Rieben R. Dextran sulfate acts as an endothelial cell protectant and inhibits human complement and NK cell-mediated cytotoxicity against porcine cells. Transplantation 2003 2003; 76:838-843.

23. Zeerleder S, Mauron T, Lammle B, Wuillemin WA. Effect of low-molecular weight dextran sulfate on coagulation and platelet function tests. Thromb Res 2002;105:441-446.

24. Dodge HT, Sandler H, Ballew DW, Lord JD Jr. The use of biplane angio cardigraphy for the measurement of left ventricular volume in man. Am Heart J 1960;60:762-776.

25. Klein HH, Puschmann S, Schaper J, Schaper W. The mechanisms of the tetrazolium reaction in identifying experimental myocardial infarction. Virchows Arch 1981;393:287-297.

26. Daha MR, Van Es LA. Enhanced alternative complement pathwaydependent degradation of soluble immunoglobulin aggregates by macrophages. Immunology 1981;43:513-518.

27. Thiruvikraman SV, Guha A, Roboz J, Taubman MB, Nemerson Y, Fallon JT. In situ localization of tissue factor in human atherosclerotic plaques by binding of digoxigenin-labeled factors VIla and X. Lab Invest 1996; 75:451-461.
28. Gollackner B, Goh SK, Qawi I, Buhler L, Knosalla C, Daniel S, Kaczmarek E, Awwad M, Cooper DK, Robson SC. Acute vascular rejection of xenografts: roles of natural and elicited xenoreactive antibodies in activation of vascular endothelial cells and induction of procoagulant activity. Transplantation 2004;77:1735-1741.

29. Friedrichs GS, Kilgore KS, Manley PJ, Gralinski MR, Lucchesi BR. Effects of heparin and $\mathrm{N}$-acetyl heparin on ischemia/reperfusion-induced alterations in myocardial function in the rabbit isolated heart. Circ Res 1994; 75:701-710.

30. Lauver DA, Booth EA, White AJ, Poradosu E, Lucchesi BR. Sulodexide (KRX-101) attenuates myocardial ischemia/reperfusion injury and the deposition of C-reactive protein in areas of infarction without affecting hemostasis. J Pharmacol Exp Ther 2005;312:794-800.

31. Black SC, Gralinski MR, Friedrichs GS, Kilgore KS, Driscoll EM, Lucchesi BR. Cardioprotective effects of heparin or $\mathrm{N}$-acetylheparin in an in vivo model of myocardial ischaemic and reperfusion injury. Cardiovasc Res 1995;29:629-636.

32. Pinckard RN, O'Rourke RA, Crawford MH, Grover FS, McManus LM, Ghidoni JJ, Storrs SB, Olson MS. Complement localization and mediation of ischemic injury in baboon myocardium. J Clin Invest 1980;66: 1050-1056.

33. Weisman HF, Bartow T, Leppo MK, Marsh HC Jr., Carson GR, Concino MF, Boyle MP, Roux KH, Weisfeldt ML, Fearon DT. Soluble human complement receptor type 1: in vivo inhibitor of complement suppressing postischemic myocardial inflammation and necrosis. Science 1990;249: 146-151.

34. Pratt JR, Jones ME, Dong J, Zhou W, Chowdhury P, Smith RA, Sacks SH. Nontransgenic hyperexpression of a complement regulator in donor kidney modulates transplant ischemia/reperfusion damage, acute rejection, and chronic nephropathy. Am J Pathol 2003;163:1457-1465.

35. de Zwaan C, Kleine AH, Diris JH, Glatz JF, Wellens HJ, Strengers PF, Tissing M, Hack CE, van Dieijen-Visser MP, Hermens WT. Continuous 48-h C1-inhibitor treatment, following reperfusion therapy, in patients with acute myocardial infarction. Eur Heart J 2002;23:1670-1677.

36. Rossen RD, Swain JL, Michael LH, Weakley S, Giannini E, Entman ML. Selective accumulation of the first component of complement and leukocytes in ischemic canine heart muscle. A possible initiator of an extra myocardial mechanism of ischemic injury. Circ Res 1985;57:119-130.

37. Kagiyama A, Savage HE, Michael LH, Hanson G, Entman ML, Rossen RD. Molecular basis of complement activation in ischemic myocardium: identification of specific molecules of mitochondrial origin that bind human C1q and fix complement. Circ Res 1989;64:607-615.

38. Birdsall HH, Green DM, Trial J, Youker KA, Burns AR, MacKay CR, LaRosa GJ, Hawkins HK, Smith CW, Michael LH, Entman ML, Rossen RD. Complement C5a, TGF-beta 1, and MCP-1, in sequence, induce migration of monocytes into ischemic canine myocardium within the first one to five hours after reperfusion. Circulation 1997;95:684-692.

39. de Vries B, Walter SJ, Peutz-Kootstra CJ, Wolfs TG, van Heurn LW, Buurman WA. The mannose-binding lectin-pathway is involved in complement activation in the course of renal ischemia-reperfusion injury. Am J Pathol 2004;165:1677-1688.

40. Jordan JE, Montalto MC, Stahl GL. Inhibition of mannose-binding lectin reduces postischemic myocardial reperfusion injury. Circulation 2001; 104:1413-1418.

41. Esmon CT. Thrombomodulin as a model of molecular mechanisms that modulate protease specificity and function at the vessel surface. Faseb J 1995;9:946-955.

42. Marcum JA, Atha DH, Fritze LM, Nawroth P, Stern D, Rosenberg RD. Cloned bovine aortic endothelial cells synthesize anticoagulantly active heparan sulfate proteoglycan. J Biol Chem 1986;261:7507-7517.

43. Chen D, Giannopoulos K, Shiels PG, Webster Z, McVey JH, Kemball-Cook G, Tuddenham E, Moore M, Lechler R, Dorling A. Inhibition of intravascular thrombosis in murine endotoxemia by targeted expression of hirudin and tissue factor pathway inhibitor analogs to activated endothelium. Blood 2004; 104:1344-1349.

44. Chou J, Mackman N, Merrill-Skoloff G, Pedersen B, Furie BC, Furie B. Hematopoietic cell-derived microparticle tissue factor contributes to fibrin formation during thrombus propagation. Blood 2004;104: 3190-3197.

45. Ruf W, Edgington TS. Structural biology of tissue factor, the initiator of thrombogenesis in vivo. Faseb J 1994;8:385-390.

46. Golino P, Ragni M, Cirillo P, Scognamiglio A, Ravera A, Buono C, Guarino A, Piro O, Lambiase C, Botticella F, Ezban M, Condorelli M, Chiariello M. Recombinant human, active site-blocked factor VIla reduces infarct size and no-reflow phenomenon in rabbits. Am J Physiol Heart Circ Physiol 2000;278:H1507-H1516. 
47. Horstick G, Heimann A, Gotze O, Hafner G, Berg O, Boehmer P, Becker P, Darius H, Rupprecht HJ, Loos M, Bhakdi S, Meyer J, Kempski O. Intracoronary application of $\mathrm{C} 1$ esterase inhibitor improves cardiac function and reduces myocardial necrosis in an experimental model of ischemia and reperfusion. Circulation 1997;95:701-708.

48. Vakeva AP, Agah A, Rollins SA, Matis LA, Li L, Stahl GL. Myocardial infarction and apoptosis after myocardial ischemia and reperfusion: role of the terminal complement components and inhibition by anti-C5 therapy. Circulation 1998;97:2259-2267.

49. Solomon SD, Glynn RJ, Greaves S, Ajani U, Rouleau JL, Menapace F, Arnold JM, Hennekens C, Pfeffer MA. Recovery of ventricular function after myocardial infarction in the reperfusion era: the healing and early afterload reducing therapy study. Ann Intern Med 2001;134: 451-458.

50. Antoniucci D, Valenti R, Migliorini A, Moschi G, Trapani M, Buonamici P, Cerisano G, Bolognese L, Santoro GM. Relation of time to treatment and mortality in patients with acute myocardial infarction undergoing primary coronary angioplasty. Am J Cardiol 2002;89:1248-1252.

51. De Luca G, van't Hof AW, de Boer MJ, Ottervanger JP, Hoorntje JC, Gosselink AT, Dambrink JH, Zijlstra F, Suryapranata H. Time-to-treatment significantly affects the extent of ST-segment resolution and myocardial blush in patients with acute myocardial infarction treated by primary angioplasty. Eur Heart J 2004;25:1009-1013.
52. Garcia-Dorado D, Theroux P, Elizaga J, Galinanes M, Solares J, Riesgo M, Gomez MJ, Garcia-Dorado A, Fernandez Aviles F. Myocardial reperfusion in the pig heart model: infarct size and duration of coronary occlusion. Cardiovasc Res 1987;21:537-544.

53. Reimer KA, Vander Heide RS, Richard VJ. Reperfusion in acute myocardial infarction: effect of timing and modulating factors in experimental models. Am J Cardiol 1993;72:13G-21G.

54. Maxwell MP, Hearse DJ, Yellon DM. Species variation in the coronary collateral circulation during regional myocardial ischaemia: a critical determinant of the rate of evolution and extent of myocardial infarction. Cardiovasc Res 1987;21:737-746.

55. Schaper W, Frenzel H, Hort W. Experimental coronary artery occlusion. I. Measurement of infarct size. Basic Res Cardiol 1979;74:46-53.

56. Ito WD, Schaarschmidt S, Klask R, Hansen S, Schafer HJ, Mathey D, Bhakdi S. Infarct size measurement by triphenyltetrazolium chloride staining versus in vivo injection of propidium iodide. J Mol Cell Cardiol 1997; 29:2169-2175

57. Wolff RA, Chien GL, van Winkle DM. Propidium iodide compares favorably with histology and triphenyl tetrazolium chloride in the assessment of experimentally-induced infarct size. J Mol Cell Cardiol 2000;32:225-232.

58. van den Berg BM, Vink H, Spaan JA. The endothelial glycocalyx protects against myocardial edema. Circ Res 2003;92:592-594.

59. Mulivor AW, Lipowsky HH. Role of glycocalyx in leukocyte-endothelial cell adhesion. Am J Physiol Heart Circ Physiol 2002;283:H1282-H1291. 